Conclusion: The daily team leader report was considered critical to understanding how each team member was responding to the environment during the TC Winston response. It prompted strategies to manage heat and hydration prior to the manifestation of serious symptoms. Such information also contributed to our knowledge of the AusMAT member workloads, assisting to prepare teams for future deployments. ${ }^{1}$ Brearley M, Ruskie S. Development of a Disaster Nurse Well-being Instrument. Prehospital and Disaster Medicine 30(1): s116

Prehosp Disaster Med 2017;32(Suppl. 1):s111-s112

doi:10.1017/S1049023X1700320X

\section{Canada Task Force 2 Medical Team Deployment to the} Fort McMurray Wildfire

Joshua Bezanson ${ }^{1}$, Gwynn Curran-Sills ${ }^{2}$, Dirk Chisholm ${ }^{3}$, Kevin Hanrahan $^{4}$

1. Emergency Medicine, University of Alberta, Edmonton/AB/Canada

2. Family Medicine, University of Calgary, Calgary/AB/Canada

3. Canada Task Force 2 Calgary/AB/Canada

4. Emergency Medicine, University of Calgary, Calgary/AB/Canada

Study/Objective: To provide a review of the Canada Task Force 2 (CAN-TF2) medical team deployment to the 2016 Fort McMurray wildfire (FMMW).

Background: The FMMW displaced over 80,000 citizens and destroyed over 1,600 structures, making it the most economically devastating natural disaster in Canadian history, with a cost of over 9 billion CDN (6,834 billion US). CAN-TF2, an all-hazards disaster response team, was deployed to this disaster. Since its inception in 2002, the team has deployed in Alberta during the Slave Lake wildfires (2011), and the Calgary/High River floods (2013). The medical unit of CAN-FT2 engaged in its first active deployment during the FMMW. This team was designed to provide medical care for CAN-FT2 members and was comprised of paramedics, nurses, and physicians. During this event the teams scope was expanded, as it developed the only medical facility in the FMM vicinity.

Methods: A narrative review of the FMMW deployment that focused on lessons learned from the medical team standpoint, along with descriptive epidemiology of the patient encounters. Results: Themes discussed included: (1) the development of a field hospital to support those beyond the CAN-FT2 team, which encompassed first-responders and critical infrastructure employees; (2) undertaking chronic disease management; (3) the fostering of relationships with other provincial agencies that allowed access to medical transport and critical medical supplies; (4) the integration of a critical incident stress-management team that addressed the mental health needs of first-responders; (5) the monitoring of public health markers and advocacy for actions within the incident command structure, that ensured the safety of the first-responders and self-deployed volunteers; (6) the transition from a CAN-TF2 field hospital back to a government facility run by the local medical community in FMM.

Conclusion: The medical team capacity within CAN-FT2 continues to evolve, and the FMMW deployment has highlighted a number of strengths and areas requiring further development.

Prehosp Disaster Med 2017;32(Suppl. 1):s112

doi:10.1017/S1049023X17003211
Fit for Duty? The Case for Disaster Responder Fitness Standards Matt Brearley, Abigail Trewin

Disaster Response and Preparedness, National Critical Care and Trauma Response Centre, Darwin/NT/Australia

Study/Objective: To mitigate the risk of disaster responders suffering heat illness through physical fitness standards.

Background: Recent Australian Medical Assistance Team (AusMAT) deployments have encountered challenging environmental conditions, heightening the risk of heat stress impacting responders. Two intrinsic factors increasing the risk of heat related illness are inadequate heat acclimatisation and lower levels of physical fitness. Pre-deployment heat acclimatization guidelines have been developed for disaster responders ${ }^{1}$, yet commensurate standards are not available for fitness. Furthermore, the physiological impost of responding to disasters in hot and humid conditions are poorly characterized, limiting the development of evidence based standards.

Methods: A literature review of emergency responder fitness standards was conducted. Assessment of disaster responders was undertaken according to Brearley et al. $(2013)^{2}$ during construction of an EMT2 facility in hot and humid conditions to determine physiological and perceptual responses.

Results: Fitness standards are common among law enforcement and civilian protection agencies, generally incorporating running to volitional exhaustion. There were no reports of fitness standards for medical disaster responders in the peerreviewed literature. Establishing an EMT2 facility in hot and humid conditions resulted in prolonged elevation of heart rate, core temperature, and physiological strain accompanied by body temperature perceptions of warm to very hot.

Conclusion: Based upon the physiological responses of disaster responders establishing an EMT2 facility in hot and humid conditions, assessment of disaster responder fitness is warranted. Reflecting the lower physical demands compared to law enforcement and civilian protection agencies, and age range of potential disaster responders, submaximal fitness tests should be prioritized.

References

1. Brearley M. Pre-deployment heat acclimatization guidelines for disaster responders. Prehospital and Disaster Medicine 31(1):85-89, 2016.

2. Brearley M, Heaney M, Norton I. Physiological responses of medical team members to a simulated emergency in tropical field conditions. Prehospital and Disaster Medicine 8(2):139-144, 2013.

Prehosp Disaster Med 2017;32(Suppl. 1):s112

doi:10.1017/S1049023X17003223

Earthquake in Amatrice (Italy), August 24, 2016: The Role of the Medical Teams of the National Alpine Rescue Corp (CNSAS)

Francesco Foti ${ }^{1}$, Mario Milani ${ }^{2}$

1. Aat Como, Azienda Regionale Emergenza Urgenza Regione Lombardia, Villa Guardia/Italy

2. Snamed, CNSAS, Lecco/Italy

Study/Objective: The Mountain and Speleological Alpine Rescue Corp (CNSAS) is a voluntary corps diffused in all of Italy, expert in hostile environment rescue missions. The study is a report of the first medical teams deployed in Amatrice. 